\title{
Review of: "Climatic windows for human migration out of Africa in the past 300,000 years"
}

Hui Li ${ }^{1}$

1 Fudan University

Potential competing interests: The author(s) declared that no potential competing interests exist.

\section{Perfect Match between genealogy and climate for modern human origin}

\section{Hui Li}

MOE Key Laboratory of Contemporary Anthropology, Fudan University, Shanghai 200438, China

The Out-of-Africa dispersal of modern human was proved by evidences from both genetics and archaeology. However, evidences from more other disciplines will still be helpful in strengthening the hypothesis and revealing details of the dispersal. The climate change during the evolution of modern human was even essential in understanding the dispersal. The paper titled "Climatic windows for human migration out of Africa in the past 300,000 years" by Beyer et al. provided a very detailed dataset of rainfall in northern Africa around the time of "Out of Africa". It is even more commendable that the authors revealed that the hunter-gatherers cannot live with precipitation lower than $90 \mathrm{~mm} / \mathrm{year}$ by investigating modern populations in Africa. The result was then employed to judge the possibility of the route for early modern human to migrate out of Africa. This was what they called "Climatic Windows", an important concept for human evolution. By reading this paper, plenty of new knowledges were added in human evolution discipline, while more conclusions can be drawn if the authors would have employed slightly more precise genetic genealogy.

\section{Matching the routes with the divergences of genetic phylogeny}

As we all acknowledged, the modern human originated in East Africa, and therefore, we mostly take it as granted that early modern human migrated out of Africa from the northeastern side, and Sinai route was the first option in most introductions of human origin study. However, there had never been any assessment for the possibility of human migration through this route on climate aspect before this study, which might be the most important. This study gave very convinced evidences that the Mandab route was much more suitable for human to go through than the Sinai route around 60-70 thousand years ago. Certainly, there would still be possible for early human migrated along Nile River to Sinai Peninsula into West Asia. More critical opinion will be that one should not only assess the northeastern routes as there 
were more routes connecting Africa to Eurasia during the Last Glacial Epoch, such as the Gibraltar route and Sicily route. If the team provided the analyses included these two more routes, which, I believe, will support the present hypothesis perfectly, the story would be much more comprehensive.

Moreover, the Mandab route, which the authors called the southern route, can explain the early divergences of the $Y$ chromosome phylogeny much better than any other routes. Because the efficient population size of $Y$ chromosome is much smaller than that of other genetic materials, it drifted to most different haplotypes during early human migration. Sometimes that would be different in every route. We found that the $\mathrm{Y}$ phylogeny diverged into two branches, i.e., DE and CF, as soon as they migrated out of Africa. That means there were two routes in front of the migrants when they stepped on the land of Asia. The square shape of Arabian Peninsula met the condition. The haplogroup DE might have been carried to the north and CF to the east. This will be the simplest explain for the genetic divergence, while there would still be possible that $D E$ have gone along Nile to the north and then diverged again into the west $E$ in West Africa and east D in Southeastern Asia. We have now found the most ancient haplogroup DE before further divergence in West Asia, which made us believe that DE was most probably carried through Mandab route. These discusses combining climate and genetics will enhance the conclusion of the paper.

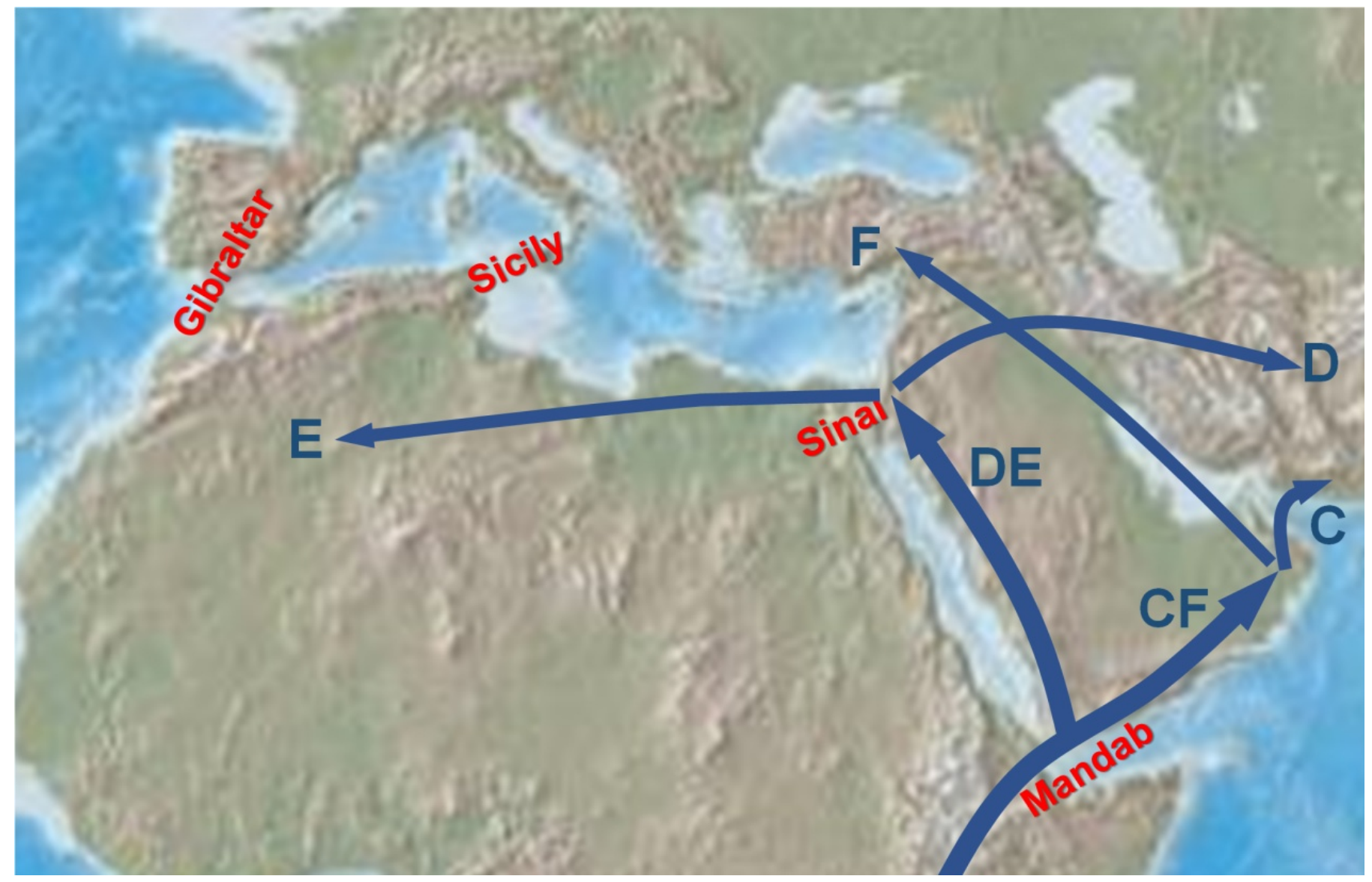

Fig.1. Possible routes out of Africa and Y chromosome phylogeny fitted into map. 


\section{More precise time of modern human evolution}

The authors analyzed five time points, 246k, 230k, 130k, 90k, and 64k years ago, which were all helpful in understanding the evolution period of modern human. However, we will be most interested in seeing the climates of $195 \mathrm{k}$ and $70 \mathrm{k}$ year ago, which might be the time of the emergence of modern human and the time of Out-of-Africa judging from the genetic phylogeny. Moreover, the earliest fossil of modern human found in Omo, Ethiopia dated to 195k years ago, made this time much more important in modern human origin. Judged from the trends given in the paper, climate changed dramatically around 195k years ago. That needs to be noticed by all relevant researchers.

The other essential time was $74 \mathrm{k}$ years ago, the time of Toba eruption, which was shown very clearly in the climate change trends in this paper. The eruption of Toba volcano in Sumatra changed the climate of the entire earth dramatically, and some call that Toba Catastrophe. Toba eruption might be the main cause of the Last Glacial Maximum, and largely reduced the populations of most animals and plants on the earth, including Neanderthals and Denisovans, as well as modern human. Fortunately, modern human lived in Africa quite far from Sumatra, and not as cold as Eurasia in Last Glacial Maximum, and therefore the population grew much quicker than Neanderthals and Denisovans. Soon, several thousand years after the eruption, they dispersed into West Asia. Thus, the time of Out-of-Africa might not as late as 64k but some age around 70k years ago. To mention this background will make this paper more interesting, and explain the data they presented much better.

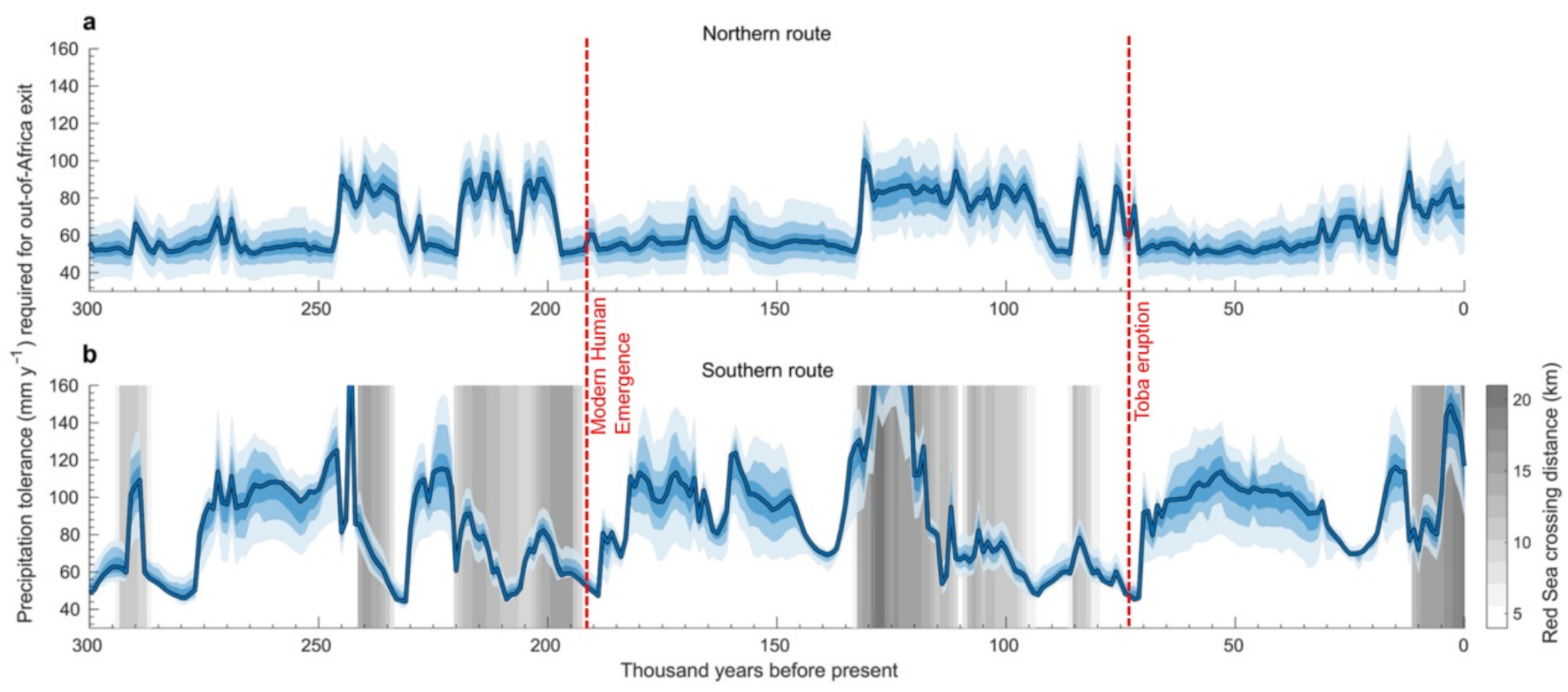

Fig.2. Ages of the earliest modern human and the Out-of-Africa matches the climate change perfectly.

Overall, this is a really perfect work. I believe that every relevant researcher can find his interest and read 
out more information for better understanding the human evolution. 